Herz 2011 · 36:667-668

DOI 10.1007/s00059-011-3537-1

Online publiziert: 4. November 2011

(c) Urban \& Vogel 2011

\author{
H. Jakob \\ Klinik für Thorax- und Kardiovaskuläre Chirurgie, \\ Westdeutsches Herzzentrum Essen, Essen
}

\title{
Was gibt es Neues in der Herzchirurgie?
}

Mit Freude habe ich den Auftrag angenommen, ein Heft mit dem Titel „Was gibt es Neues in der Herzchirurgie“ zusammenzustellen. In Kontinuität zum Heft 5/o6 gelang es weitgehend, mit den damals beteiligten herzchirurgischen Gruppen erneut zusammenzuarbeiten. Es wurde großer Wert darauf gelegt, die Artikel übersichtlich und kompakt für Nichtchirurgen zu verfassen, um bei geringem Zeitaufwand einen Überblick über den aktuellen Stand der herzchirurgischen Verfahren und deren Ergebniskultur zu geben.

Mit rund 56.00o Eingriffen stellt die Koronarchirurgie nach wie vor die größte Subgruppe in der Deutschen Herzchirurgie dar. Durch die SYNTAX-Studie mit ihren jetzt vorliegenden Dreijahresergebnissen existiert erstmals ein weltweit anerkanntes Vergleichsinstrument, basierend auf randomisierten "All-comers“Daten zwischen Bypass-Operation und PCI, was die Kieler Arbeitsgruppe um Jochen Cremer in ihrem Artikel analysiert, die zu dem Schluss kommt, dass die koronare Bypass-Operation unvermindert den „Goldstandard“ bei fortgeschrittener Dreigefäßerkrankung und vor allem deren koronaranatomisch komplexen Formen sowie bei Hauptstammstenose darstellt. An die in den europäischen Leitlinien geforderte Schaffung sog. HerzTeams, bestehend aus Kardiologen und Herzchirurgen, mit nachfolgender gemeinsamer Diskussion mit dem Patienten hinsichtlich seiner individuell $\mathrm{zu}$ wählenden bestmöglichen Therapieoption wird erinnert.

Während die Mitralklappenrekonstruktion etwa $65 \%$ aller Mitralklappen- eingriffe in Deutschland ausmacht und an einigen herzchirurgischen Zentren die minimal-invasive, endoskopisch assistierte Variante mit ihrem deutlich reduzierten Operationstrauma und exzellenten Langzeitresultaten zum Verfahren der Wahl wurde, entwickeln sich katheterbasierte, perkutane Verfahren als veritable Alternativansätze bei älteren Patienten mit deutlich eingeschränkter Ventrikelfunktion und funktioneller Mitralklappeninsuffizienz, wie die Autoren des Hamburger Universitären Herzzentrums berichten. Derzeit ist jedoch lediglich das MitraClip -Verfahren im klinischen Einsatz; die Koronarvenensinusanuloplastie ist verlassen.

Die adjunktive chirurgische Therapie des assoziierten Vorhofflimmerns bei Vitien- oder Koronaroperationen hat entsprechend den 2010 erschienenen Richtlinien der Europäischen Gesellschaft für Kardiologie den Status „Evidenzklasse IIa, Level A“ erhalten. Das alleinige (symptomatische) Vorhofflimmern ist jedoch zweifelsohne eine Domäne der Kardiologie. Interventionelle Ablationsverfahren stellen hier das Verfahren der Wahl dar. Im Fall eines Therapieversagens (bis zu $30 \%$ ) sind jedoch die chirurgischen, minimal-invasiven Kryo- oder bipolaren endokardialen Verfahren eine sinnvolle und mit guten Ergebnissen vergesellschaftete Alternative, wie die Lübecker Arbeitsgruppe um Hans Sievers berichtet.

Die Essener Arbeitsgruppe gibt einen Überblick über die rasch wachsenden Transkatheteraortenklappenimplantationen und die derzeit vorhandenen oder in Entwicklung befindlichen Transkathetersysteme, die darauf abzielen, Nachtei- le oder Schwächen der Erst- und Zweitgenerationssysteme zu überwinden.

Schließlich wird in einem weiteren Artikel über interessante Entwicklungen zur Erhöhung der Implantationssicherheit von Stent-Graft-Prothesen in der thorakalen Aortenchirurgie berichtet, indem angioskopische Verfahren mit direkter Visualisierung der Aortenpathologie zum Einsatz kommen.

Allen Artikeln gemeinsam ist die Demonstration einer gelebten und noch weiter auszubauenden Interdisziplinarität zwischen Herzchirurgen und Kardiologen, die zunehmend zur Schaffung von Herz-, Klappen-, Rhythmus- und Aorten-Teams führt, mit Einbringung der Expertise beider Fachdisziplinen und der gemeinsamen Beschlussfassung und Durchführung von Eingriffen, z. B. in gemeinsamen Hybridräumen. Diese Überwindung von formalen Fachgrenzen ist eine von uns allen gewollte, sehr positive Perspektive.

\section{Heinz Jakob, Essen}

\section{Korrespondenzadresse}

Prof. Dr. H. Jakob

Klinik für Thorax- und Kardiovaskuläre Chirurgie, Westdeutsches Herzzentrum Essen Hufelandstraße 55, 45122 Essen

heinz.jakob@uk-essen.de 


\section{Hier steht eine Anzeige.}

Springer 